\title{
O.S.P.
}

\section{Le rôle des relations socio-émotionnelles dans les transitions scolaires et professionnelles}

The role of socioemotional relationships in school and work transition

Donna E. Palladino Schultheiss

Traducteur : Alain Frangi

(2) OpenEdition

Journals

\section{Édition électronique}

URL : https://journals.openedition.org/osp/659

DOI : 10.4000/osp.659

ISSN : 2104-3795

Éditeur

Institut national d'étude du travail et d'orientation professionnelle (INETOP)

\section{Édition imprimée}

Date de publication : 1 septembre 2005

Pagination : 353-373

ISSN : 0249-6739

Référence électronique

Donna E. Palladino Schultheiss, « Le rôle des relations socio-émotionnelles dans les transitions scolaires et professionnelles », L'orientation scolaire et professionnelle [En ligne], 34/3 | 2005, mis en ligne le 28 septembre 2009, consulté le 18 janvier 2022. URL : http://journals.openedition.org/osp/659 ; DOl : https://doi.org/10.4000/osp.659

Ce document a été généré automatiquement le 18 janvier 2022.

(c) Tous droits réservés 


\title{
Le rôle des relations socio- émotionnelles dans les transitions scolaires et professionnelles
}

The role of socioemotional relationships in school and work transition

\author{
Donna E. Palladino Schultheiss
}

Traduction : Alain Frangi

\section{NOTE DE L'ÉDITEUR}

La version anglaise de ce manuscrit a été traduite par Alain Frangi.

Dans un climat de changement mondial accéléré, il n'est pas surprenant que les transitions, à l'école comme au travail, créent de l'anxiété chez les adolescents et les adultes qui les vivent. Dans un monde devenu moins sûr et moins prévisible, ces transitions impliquent des remises en cause potentiellement anxiogènes pour tous les habitants du globe. Les transitions au cours de la vie constituent des phases très sensibles où l'interaction de forces sociostructurelles, institutionnelles et individuelles fournit l'occasion d'un développement et d'une ascension sociale, mais aussi un risque de déclin et de spirale descendante. Si les nations diffèrent quant à la structuration sociétale de ces transitions (par exemple, la transition de l'école au travail) et dans leur potentiel global de mobilité sociale, on peut néanmoins trouver des aspects universels de l'expérience humaine qui transcendent les limites nationales. Les réseaux interpersonnels qui sous-tendent les contacts sociaux fournissent le contexte imbriqué où les transitions ont lieu, à l'école et au travail. Le contexte relationnel peut faire s'épanouir un semis fertile ou aussi laisser en friche le terrain sur lequel le développement individuel s'opère dans le contexte éducatif et professionnel. Bien qu'on ne puisse examiner les relations isolément d'autres facteurs imbriqués dans le contexte comme l'environnement historique, sociopolitique et économique, les publications dans le champ de l'orientation professionnelle offrent de nombreuses 
perspectives sur les influences relationnelles. Cet article a pour objet l'importance et la fonction de la relation interpersonnelle (en particulier des relations familiales) chez des adolescents et jeunes adultes engagés dans des défis tels que ceux qu'on rencontre lors des transitions de l'école au travail.

Les investigations actuelles des approches relationnelles pour comprendre et faciliter les transitions ont leurs racines dans un cadre historique et théorique plus vaste (Erikson, 1968; Youniss \& Smollar, 1985). La nouveauté concernant les approches relationnelles contemporaines présentées ici, c'est une perspective postmoderne offrant un cadre conceptuel holistique pour comprendre l'influence multidimensionnelle des relations familiales. À ce jour, personne n'a proposé d'historique complet de cet ensemble de travaux, ni organisé celui-ci de façon à donner tout leur sens à ses concepts. Étant donné que la période exploratoire entre adolescence et âge adulte s'allonge pour un nombre toujours plus important d'individus dans le monde, un ancrage de ces travaux dans un cadre conceptuel et historique pourrait aider à formuler certains des problèmes posés par la transition de l'école vers le travail. Donc, le but de cet article est de faire avancer ce domaine de la recherche vers une compréhension des problèmes rencontrés par des individus de plus en plus nombreux partout dans le monde qui prenne pleinement la mesure des facteurs contextuels, ce qui suppose de : 1) placer ce corpus de travaux dans le cadre théorique, conceptuellement riche, de "l'âge de l'adulte en émergence " (Arnett, 2000) et 2) proposer une évaluation critique de l'état actuel des travaux théoriques et empiriques traitant des relations interpersonnelles et des transitions scolaires et professionnelles.

Les travaux sur les relations interpersonnelles et les transitions à l'école et au travail sont d'abord présentés dans le cadre des avancées récentes de la psychologie du développement qui a introduit le concept de «l'adulte en émergence » pour représenter l'essence de la période développementale qui fait le lien entre l'adolescent et l'adulte. Suit la présentation d'un bref aperçu des transitions scolaires et professionnelles. Puis on trouvera une revue critique des perspectives théoriques et empiriques (historiques et contemporaines) sur les relations interpersonnelles et les progrès de la carrière ainsi que sur les transitions scolaires et professionnelles. Enfin, une discussion sur les implications pour la recherche à venir et la pratique du conseil sera proposée.

\section{L'âge de l'adulte en émergence}

4 Le concept d'âge de l'adulte en émergence fournit un modèle utile pour cadrer la période entre 18 et 25 ans au cours de laquelle ont lieu la plupart des transitions écoletravail (Arnett, 2000). Cette période développementale entre adolescence et âge adulte se caractérise par le changement et l'exploration des directions possibles et variées que peut prendre une vie en ce qui concerne l'amour, le travail et la façon de voir le monde. Cette période est particulièrement marquée dans les cultures permettant une période d'exploration prolongée dans les années situées autour de l'âge de vingt ans. Arnett décrit l'âge de l'adulte en émergence comme une période développementale relativement indépendante des rôles sociaux et des attentes normatives. Les explorations se font alors en partie pour elles-mêmes, avant la prise de responsabilités d'adulte durables et limitatives. Pour la plupart des jeunes, dans les pays industrialisés, on définit cette période comme distincte démographiquement, subjectivement et en 
termes d'exploration de l'identité (Arnett, 2000). Elle se caractérise par un niveau élevé de diversité démographique et d'instabilité, car l'individu risque moins d'être contraint de tenir un rôle dans cette période développementale. Par exemple, le statut résidentiel (c'est-à-dire, habiter chez ses parents, seul, ou avec quelqu'un d'autre) et le statut éducatif (c'est-à-dire, inscription ou non dans un établissement d'enseignement supérieur) font partie de ces variables démographiques reflétant le statut exploratoire de cette période. À vrai dire, l'hétérogénéité de cette période développementale est l'un des traits les plus caractéristiques de l'âge de l'adulte en émergence. Les caractéristiques subjectives qui semblent les plus importantes dans l'impression qu'a un individu d'atteindre l'âge adulte sont: accepter d'être responsable de soi-même, prendre des décisions indépendantes et conquérir son autonomie financière. Les explorations identitaires se placent dans trois domaines principaux : l'amour, le travail et la vision du monde. À l'âge où l'adulte est en émergence, il semble que les explorations de l'amour deviennent plus intimes et sérieuses, celles du travail moins éphémères et provisoires, mais plus sérieuses et affinées, et qu'on examine et envisage diverses visions du monde.

5 Cette période de la vie au cours de laquelle, de façon prolongée et progressive, l'adulte est en émergence n'est pas une phase universelle qu'on trouverait dans toutes les cultures, mais plutôt une période qui n'existe que dans les cultures repoussant l'entrée dans les rôles et responsabilités adultes bien au-delà de l'adolescence (Arnett, 2000). On a donc plus de chances de la trouver en tant que phase développementale dans les pays industrialisés, en partie à cause du haut niveau d'études requis pour accéder aux professions impliquant de grandes compétences techniques. Pourtant, d'après Arnett elle pourrait s'étendre à toute la planète en tant que période de développement à mesure que la mondialisation touche les diverses économies du monde. Cette période de la vie présente mille possibilités et un potentiel de transition vers autant de futurs possibles. Pour la plupart des gens, la liberté et l'exploration personnelle sont plus grandes à ce moment qu'à n'importe quel autre (Arnett, 2000). Ainsi le concept d'adulte en émergence est utile pour rendre compte des transitions école-travail qui se produisent surtout à cette période du développement.

\section{Les transitions scolaires et professionnelles}

6 La transition école-travail des jeunes qui ne font pas d'études supérieures attire de plus en plus l'attention des chercheurs du monde entier (Blustein, Juntunen \& Worthington, 2000 ; Bynner, 1998 ; Feij, Whitely, Peiro \& Taris, 1995 ; Heckhausen, 2002). C'est la période commençant avant la fin des études secondaires et se poursuivant après l'acquisition d'un premier travail. Ses limites ne sont donc pas l'arrêt des études et l'obtention d'un emploi; au contraire, elle inclut la période développementale qui précède l'arrêt des études et continue jusqu'à l'indépendance financière de l'individu (Phillips, Blustein, Jobin-Davis \& White, 2002 ; Reitzle, Vondracek \& Silbereisen, 1998). De toutes les transitions développementales, c'est l'entrée dans le monde du travail qui est considérée comme la plus centrale dans la formation de l'identité adulte (Bynner, 1998 ; Savickas, 1993). Le rôle critique du contexte dans la formation de l'identité est devenu encore plus évident dans notre société mondialisée. Dans le contexte d'un marché du travail fluide et en pleine évolution, les individus sont contraints de mettre leur identité en conformité avec les circonstances changeantes de ce marché. On a donc 
proposé de nommer ces périodes «navigation » (dans la vie) plutôt que «transitions » (Bynner, 1998 ; Evans \& Furlong, 1996 ; Evans \& Heinz, 1994).

$7 \quad$ La plupart des chercheurs acceptent l'idée que les transitions comportent des périodes développementales critiques ayant le potentiel d'amplifier les effets des ressources différentielles, sociales et individuelles, pour l'avenir de l'individu (Heckhausen, 2002). Ceci est en particulier vrai pour la transition école-travail, qui propulse l'adolescent dans sa propre carrière et sa propre famille en tant qu'adulte. Ainsi, la transition écoletravail a des conséquences particulièrement significatives sur plusieurs domaines dont ceux qui sont en relation avec le bien-être psychologique et économique de l'individu et la capacité de la société à promouvoir les talents et les ressources de ses membres (Heckhausen, 2002). Les nations présentent des différences considérables en ce qui concerne leur structuration sociale de cette transition, sous plusieurs aspects majeurs : 1) le degré auquel la transition est prédéterminée par des institutions éducatives établies, les stages professionnels et les modes d'entrée sur le marché du travail ; 2) le degré auquel l'adolescent peut influencer la transition et doit jouer un rôle actif ; et 3) par rapport aux conséquences de cette transition sur les perspectives d'emploi à long terme de l'individu (Heckhausen, 2002). Plus la transition est institutionnalisée (par exemple, le système allemand et sa dualité apprentissage/éducation formelle), moins les ressources individuelles non académiques (le réseau des relations sociales de la familles, etc.) ont d'influence sur le processus. Ainsi, il se peut qu'un système caractérisé par une sous-régulation (par exemple, aux États-Unis) agisse en conservant et en amplifiant les inégalités sociales, et rende les différences informelles et noninstitutionnalisées dans l'accès aux ressources, y compris les réseaux de connaissances, décisives pour l'entrée dans des parcours professionnels facilitant l'adaptation (Heckhausen, 2002). Dans ces situations, on peut s'attendre à ce que les différences individuelles de ressources personnelles et sociales s'amplifient et aboutissent à l'ascension sociale pour quelques privilégiés, et à une rétrogradation sur l'échelle sociale pour ceux qui viennent de milieux socio-économiques moins favorisés (Heckhausen, 2002).

8 Malgré les réelles conséquences développementales, socio-économiques et psychologiques des transitions école-travail, il y a d'importantes lacunes dans la connaissance et la compréhension du processus par lesquels les élèves du secondaire passent dans le monde du travail (Blustein, Juntunen \& Worthington, 2000 ; Lewis, Stone, Shipley \& Madzar, 1998; Phillips et al., 2002; Tinsley, 1995). Étant donné l'importance de cette période développementale, il est crucial d'identifier les antécédents et caractéristiques psychologiques des transitions école-travail facilitant l'adaptation (Blustein et al., 2000 ; Bynner, 1998; Phillips et al., 2002 ; Way \& Rossman, 1996a). Le choix d'une carrière s'effectue grâce à la confluence de contextes reliés entre eux, allant des plus distants, comme la situation économique, aux plus rapprochés, comme les relations interpersonnelles dans la vie d'un individu (Silbereisen, 2002; Vondracek, Lerner \& Schulenberg, 1986). En accord avec de récentes tentatives pour contextualiser le discours sur la conduite lors d'une orientation professionnelle (Vondracek et al., 1986; Young \& Collin, 1992), Blustein (1995) a mis l'accent sur l'importance du contexte rapproché de la transition école-travail en général et sur l'environnement relationnel en particulier. La connaissance des antécédents psychologiques de transitions favorisant l'adaptation est extrêmement importante pour la mise en place d'interventions de conseil, de recommandation de politiques et 
d'efforts vers une réforme de l'éducation (Phillips et al., 2002). Pourtant des notions fondées sur l'économie et le capital humain restent dominantes dans les travaux sur la transition école-travail (par exemple, Bynner, Ferri \& Shepherd, 1997 ; Wilson, 1996). La pauvreté de la recherche contemporaine sur les aspects sociaux et émotionnels des transitions laisse un vide déconcertant dans nos connaissances. Des relations interpersonnelles significatives peuvent apporter le soutien et la sécurité qui aident les individus à négocier plus efficacement les situations de stress telles celles qu'on rencontre au travail et lors du choix d'une carrière (Blustein, Prezioso \& Schulteiss, 1995). Je vais présenter maintenant les racines historiques des recherches sur les relations et le développement de l'adolescent, ainsi que les publications actuelles sur le rôle des relations interpersonnelles dans les carrières et la transition école-travail.

\section{Perspectives théoriques et empiriques sur les relations et le développement professionnel}

\section{Théories historiques contextuelles}

Dans les études classiques traditionnellement citées en psychologie du développement (par exemple, Kandel \& Lesser, 1972; Youniss \& Smollar, 1985), la structure des relations parent-adolescent, et ce en quoi elles diffèrent des relations précédentes parent-enfant et des relations d'amitié, étaient au centre des préoccupations. Par ailleurs, Erikson (1968) s'est intéressé à certaines influences parentales, en se centrant sur l'identification aux parents en tant que modèles pour faciliter le processus de développement identitaire. Dans une moindre mesure, ces chercheurs ont reconnu l'importance du soutien procuré à l'adolescent par ses parents et le rôle d'autorité qu'ils jouent dans l'adoption par l'adolescent des conduites attendues de lui par la société. Ce n'est que récemment qu'on a entrepris d'étudier les rapports entre la qualité et la signification des relations interpersonnelles, d'une part, et la formation professionnelle et le développement d'une carrière, d'autre part (voir Whiston \& Keller (2004) pour un historique). Encore actuellement, très peu de chercheurs prennent en compte le fait que la qualité des relations interpersonnelles pourrait faire partie des variables associées aux transitions école-travail réussies et facilitant l'adaptation (Phillips, Christopher-Siks \& Gravino, 2001). Le lien que la qualité et la signification des relations interpersonnelles (particulièrement dans la famille) entretiennent avec la transition de l'école au travail est en fait un domaine de recherche naissant, bien qu'important. À ce jour, l'essentiel des travaux sur la transition école-travail est étroitement consacré au rôle de la famille consistant à fournir un cadre socioéconomique à partir duquel des individus s'aventurent dans le monde du travail.

Cette arrivée d'une dimension «relations familiales» facilitant l'adaptation, dans les descriptions que font les universitaires de transitions, a beaucoup à offrir à la recherche mondiale en psychologie de l'orientation et à la communauté des praticiens. Une vision plus large de la nature complexe des parcours professionnels dans le contexte plus vaste des rôles et des transitions offre un cadre conceptuel nouveau reflétant la complexité de la vie des gens imbriquée dans des contextes socioculturels. Ces travaux sur les théories relationnelles contemporaines sont présentés maintenant. 


\section{Les théories relationnelles contemporaines}

11 Le terme "relationnel» renvoie à une tentative explicite de contextualiser un phénomène humain donné en explorant et en expliquant les dimensions interpersonnelles et intra-personnelles de la vie (Blustein et al., 2004). Les théoriciens de ce domaine affirment que les liens sont au centre du développement humain et qu'ils sont un contexte prégnant de l'expérience de soi (Gilligan, 1982 ; Jordan, Kaplan, Miller, Stiver \& Surrey, 1991 ; Josselson, 1992). Ainsi, la capacité à créer et maintenir des relations favorisant le développement est cruciale pour un bon épanouissement (Gilligan, 1982 ; Miller \& Stiver, 1997). Les perspectives relationnelles soutiennent en général l'idée que de nombreux aspects des luttes interpersonnelles et intrapersonnelles reflètent des efforts naturels de l'être humain pour se maintenir en contact avec autrui, s'affirmer, rechercher soutiens sociaux et attachements (Blustein et al., 2004).

12 Lorsqu'on les considère collectivement, les perspectives relationnelles représentent un ensemble d'idées théoriques plongeant leurs racines dans plusieurs sphères intellectuelles comprenant la psychologie du développement (par exemple, Youniss \& Smollar, 1985) et les théories systémiques familiales (par exemple, Chusid \& Cochran, 1989; Lopez \& Andrews, 1987; Patton \& McMahon, 1999), des théories psychanalytiques (par exemple, Kohut, 1977; Mitchell, 1988; Winicott, 1964) et féministes (Jordan et al., 1991).

Ainsi Youniss et Smollar (1985) ont présenté un cadre développemental fondé sur les théories de Piaget (1932) et Sullivan (1953) qui offre une perspective relationnelle enrichissante pour notre compréhension de l'adolescence dans un large contexte de vie. Deux concepts généraux définissent ce cadre théorique: la co-construction coopérative et l'individu-en-relations. La co-construction coopérative désigne la façon dont l'autorité unilatérale change dans la relation parent-enfant et se coordonne avec des comportements coopératifs. Le passage structurel de l'autorité unilatérale dans la relation parent-enfant à la structure relationnelle parent-adolescent qui inclut à la fois l'autorité unilatérale et la coopération permet plus d'indépendance à l'adolescence et un lien fondé non sur l'autorité, mais sur le respect mutuel des personnes. De plus, les adolescents recherchent les conseils de leurs parents (particulièrement en ce qui concerne leur projet professionnel et leur avenir) et sentent qu'ils peuvent s'engager avec eux dans une prise de décision coopérative. De même, le concept d'individu-enrelation rend compte de la manière dont les adolescents se posent en tant qu'individus par rapport à leurs parents tout en conservant une relation étroite avec eux. Cette conception est en cohérence avec les travaux ultérieurs de Grotevant et Cooper (1985, 1986) qui considèrent que l'adolescent développe son autonomie grâce à la coopération au sein des relations plutôt qu'en s'opposant à celles-ci ou en cherchant à s'en dégager (Piaget, 1932 ; Youniss, 1980). Certains chercheurs et praticiens ont utilisé les théories systémiques familiales pour décrire comment les rôles professionnels et les rôles extraprofessionnels s'entrecoupent (Chusid \& Cochran, 1989). Dans un axe de recherche particulièrement convaincant, on a proposé que les relations et expériences familiales se trouveraient reproduites au travail (Chusid \& Cochran, 1989; Lopez \& Andrews, 1987). Les perspectives psychanalytiques (Kohut, 1987 ; Mitchell, 1988 ; Winnicott, 1964) se concentrent sur la relation parent-enfant comme base constitutive de l'adolescent. On pense alors que la personne a été formée par cette relation et qu'elle se consolide 
ensuite par identification inconsciente aux parents. Bien qu'il y ait une grande diversité d'opinions chez les tenants de la psychanalyse, ils s'accordent généralement pour dire que la répétition des conflits à l'intérieur de la relation définit et donne forme à la relation en cours et à ses dilemmes actuels. Les universitaires féministes (par exemple, Jordan et al., 1991) affirment depuis longtemps que le sens de soi est relationnel chez la femme et que son besoin de se sentir reliée aux autres est un aspect crucial de son identité. La notion de base de la conception féministe est que la relation à autrui est le fondement de l'expérience de soi et du développement de l'identité personnelle. Dans ce modèle, il est implicite que le développement de l'identité personnelle ne nécessite aucunement qu'on lui sacrifie ou qu'on mette temporairement entre parenthèse sa vie relationnelle.

Dans toutes ces approches théoriques, la mutualité et l'interdépendance se conçoivent comme étant au cœur d'un développement et d'un fonctionnement sains. Les théories relationnelles apportent un cadre conceptuel qui facilite une compréhension plus complète de l'interdépendance de nos vies avec celles des autres.

\section{La théorie de l'attachement}

15 La théorie de l'attachement (Bowlby, 1988) propose un autre cadre théorique utile pour comprendre comment les relations fonctionnent en facilitant ou en empêchant un progrès développemental comme le passage de l'école au travail. On pense que les individus de tout âge sont mieux adaptés lorsqu'ils peuvent compter sur l'accessibilité et la capacité de réponse d'une personne de confiance. Comme le note Bowlby, l'anxiété produite par les situations nouvelles peut être atténuée par l'expérience d'un attachement à une personne importante dans la vie du sujet et pouvant apporter la sécurité émotionnelle. Cette relation, que Bowlby nomme la base sûre, peut apporter certains des ingrédients nécessaires à l'adaptation réussie aux nombreux et divers contextes nouveaux. La transition vers un nouvel environnement de travail, qui est sans nul doute encore plus compliquée pour des jeunes quittant l'école, représente le genre de défi pour lequel l'expérience d'une base sûre serait particulièrement importante (Blustein, 1995 ; Blustein et al., 1995). La théorie de l'attachement de Bowlby a servi de fondement théorique pour la recherche sur le rôle des relations dans les transitions développementales (Larose \& Boivin, 1998 ; Papini \& Roggman, 1992).

\section{Les données empiriques sur les rapports entre relations interpersonnelles et développement professionnel}

Les travaux en psychologie de l'orientation professionnelle ont vu émerger un thème d'étude marquant avec des publications de plus en plus nombreuses traitant des rapports entre la carrière et la qualité des relations de l'individu (Blustein, 2001; Blustein et al., 2004 ; Hall, 1996). Whiston et Keller (2004) ont récemment intégré de nombreuses recherches portant sur les influences de la famille sur le développement de la carrière professionnelle. Cette intégration démontre que les familles exercent bien des effets spécifiques et prévisibles sur le développement professionnel. Il est fondamental d'avoir un point de vue réaliste de l'individu comme composante d'un système familial pour comprendre comment les gens, et en particulier les adolescents, développent et mettent en œuvre des projets professionnels (Blustein, 2001 ; Savickas, 
2002a). Les recherches intégrées par Whiston et Keller (2004) suggèrent que des facteurs de relations familiales, comme les attitudes et attentes des parents vis-à-vis de l'adolescent, son identification à ceux-ci, la communication ouverte parentsadolescent, les styles éducatifs parentaux, la qualité des attachements aux parents, la séparation psychologique, l'aide et l'implication directes des parents et les aspects multidimensionnels du soutien (c'est-à-dire, le soutien émotionnel, social, instrumental et tangible, ainsi que le soutien dans le domaine de l'estime de soi et de la recherche d'information), tous ces aspects des relations exercent une influence sur l'identité professionnelle que développe l'adolescent, ses intérêts à l'égard des métiers, la maturité de sa réflexion dans ce domaine, le sentiment qu'il a d'être capable de prendre efficacement des décisions d'orientation, ses valeurs relatives au monde du travail, l'exploration qu'il fait de ses perspectives d'orientation scolaire et professionnelle et ses conduites de prise de décision dans le domaine de l'orientation (Whiston \& Keller, 2004).

17 En accord avec la théorie (Bowlby, 1988), une grande partie des recherches sur les relations familiales s'est intéressée au rôle des attachements (c'est-à-dire des relations procurant un soutien réconfortant avec des personnes qui sont pour nous significatives) dans diverses conduites concernant la carrière (Blustein et al., 1995). En particulier, on a systématiquement trouvé qu'un attachement confiant aux parents est en relation positive, chez les étudiants de premier cycle, avec plusieurs aspects de leur ajustement à la transition lycée-université. Ils explorent davantage leurs possibilités d'orientation (c'est-à-dire que le processus par lequel les individus recherchent des informations sur eux-mêmes et sur l'environnement pour prévoir et construire au mieux leur future carrière professionnelle est plus marqué) (Ketterson \& Blustein, 1997 ; Ryan, Solberg \& Brown, 1996). Ils s'engagent plus fermement dans le choix d'une profession (Blustein, Walbridge, Friedlander \& Palladino, 1991 ; Scott \& Church, 2001). Ils passent du secondaire au supérieur sans trop de problèmes sociaux, émotionnels ou académiques (Kenny \& Donaldson 1992 ; Lapsley \& Edgerton, 2002 ; Lopez \& Gormley, 2002 ; Mattanah, Hancock \& Brand, 2004 ; Schultheiss \& Blustein, 1994).

Dans un registre voisin, les résultats de Wall, Covell et MacIntyre (1999) suggèrent que les soutiens sociaux perçus par l'adolescent sont liés à ses aspirations et à ce qu'il croit pouvoir atteindre en matière de formation et de profession. Lapan, Hinkelman, Adams et Turner (1999) ont constaté que plus les élèves du secondaire s'attendent à être soutenus par leurs parents pour entreprendre certaines carrières, plus ils valorisent ces carrières. Bien que les parents semblent avoir l'influence la plus marquante sur l'orientation scolaire et professionnelle de leurs enfants, d'autres membres de la famille, comme les frères et sœurs ou des membres de la famille plus éloignés, exercent aussi ce genre d'influence importante (Schultheiss, Palma, Predragovich \& Glasscock, 2002).

19 La théorie sociale-cognitive de l'orientation professionnelle de Lent, Brown, et Hackett (1994) est à l'origine de recherches ayant souligné l'importance du soutien social comme facteur contextuel primordial dans le processus d'orientation (Lent et al., 2001 ; Lent, Brown \& Hackett, 2000). Par exemple, McWhirter, Hackett, et Bandalos (1998) ont trouvé que le soutien perçu de la part du père était associé positivement aux projets de formation et aux attentes professionnelles des lycéennes. Kenny, Blustein, Chaves, Grossman, et Gallagher (2003) se sont intéressés à la perception que les adolescents ont des obstacles s'opposant à leurs buts de formation et à la perception qu'ils ont des 
soutiens sociaux et émotionnels que leur offre leur famille. Ils ont constaté que ces deux facteurs sont liés, respectivement négativement et positivement, à l'implication scolaire des lycéens (c'est-à-dire au temps qu'ils consacrent et à l'attention qu'ils portent au travail académique), ainsi qu'à leurs aspirations à réussir professionnellement (autrement dit, à atteindre les situations les plus élevées dans un domaine choisi). Ces deux facteurs (perception des obstacles et perception des soutiens) sont liés de la même façon au fait que l'adolescent s'attend à atteindre ses buts professionnels et à l'importance qu'il accorde au travail dans la représentation qu'il se fait de son avenir. D'autres recherches ont démontré l'importance de l'influence des soutiens procurés par les parents, les amis et les enseignants, sur les attentes que les lycéens forment à l'égard de leur avenir professionnel (Paa \& McWhirter, 2000), les expériences d'enseignement positives (Fisher \& Stafford, 1999), la satisfaction au travail (Harris, Moritzen, Ribitschek, Imhoff \& Lynch, 2001) et la poursuite des buts de formation et professionnels (Juntunen et al., 2001).

\section{Stratégies de recherche actuelles concernant les rapports entre les relations interpersonnelles et l'orientation scolaire et professionnelle}

20 Le projet de mettre en place une base de connaissances pertinente qui décrive le contexte relationnel du développement de carrière en utilisant des approches qualitatives gagne du terrain (Blustein et al., 2001; Phillips et al., 2001; Schultheiss, Kress, Manzi \& Glassock, 2001 ; Schultheiss et al., 2002). Les recherches employant des méthodes quantitatives ont fait avancer notre compréhension de l'importance de la dimension relationnelle dans le développement humain. Toutefois, la nature exacte de l'influence qu'ont les relations n'a été identifiée qu'en partie. Ce qui fait défaut, c'est une exploration explicite et approfondie de la manière dont ces relations exercent leur influence (Schultheiss et al., 2001). L'approche qualitative fournit l'occasion de riches descriptions de relations et de leurs influences complexes et non-linéaires telles qu'elles se déroulent naturellement dans la vie des gens. Donc les méthodes qualitatives apportent le moyen de comprendre un phénomène grâce à un paradigme conceptuellement différent de celui qu'on peut obtenir en utilisant des approches positivistes, quantitatives et linéaires. De plus, les méthodes qualitatives fournissent une approche orientée vers la découverte, dont les résultats peuvent être utilisés dans des enquêtes quantitatives. Ce sont ces travaux que nous allons maintenant présenter.

Phillips et al. (2001) ont identifié des thèmes décrivant comment on utilise les relations lors du choix d'un métier. Ces thèmes reflétaient les manières dont les autres s'impliquaient (par exemple, en apportant leur soutien, des informations ou des alternatives), les manières dont le décideur faisait appel à leur implication (c'est-à-dire en sollicitant leur aide pour prendre une décision) et comment il les excluait de ses délibérations (c'est-à-dire en les repoussant de façon plus ou moins active). À l'aide de données qualitatives obtenues lors d'entretiens semi-structurés avec des étudiants de premier cycle et de milieu urbain, Schultheiss et al. (2001) ont identifié de multiples aspects des influences relationnelles s'exerçant sur le processus d'exploration et de prise de décision concernant leur carrière. Ces influences proviennent des parents, de la fratrie, de membres de la famille plus éloignés ou d'autres personnes significatives. Parmi les aspects de ces influences relationnelles dont le poids est le plus fort, on 
trouve le soutien social (soutien aux émotions, à l'estime de soi, sous la forme d'apport d'informations...) et le fait de fournir au jeune des modèles de rôles socioprofessionnels. D'autres facteurs relationnels importants identifiés dans cette étude étaient: des caractéristiques de personnalité ou d'ordre idéologique, les expériences durant l'enfance, les ruptures de relations, l'endroit où habitaient les parents, le fait d'avoir établi un rapport d'ordre parental avec le membre de la fratrie considéré comme le plus important, et la distance émotionnelle avec les autres frères ou sœurs.

Dans une investigation connexe, Schultheiss et al. (2002) ont examiné l'influence relationnelle réciproque des cheminements professionnels entre membres d'une même fratrie. Les résultats de cette étude soulignent l'importance, dans les conduites d'orientation, du soutien multidimensionnel, réciproque et mutuel, ainsi que des modèles de rôles professionnels perçus dans les relations avec les personnes significatives et la capacité de ces dernières à transmettre ce que leurs propres expériences professionnelles leur ont appris. Cette investigation a aussi révélé que l'influence des relations était plus forte lors des transitions scolaires et professionnelles ainsi que lors des décisions et engagements les accompagnant. La fonction essentielle du soutien relationnel, des rôles familiaux, et des comparaisons sociales lors d'une orientation scolaire et professionnelle est de plus étayée par l'analyse qualitative réalisée par Blustein et al. (2001), qui ont identifié des études de cas de conseil en orientation scolaire et professionnelle.

\section{Les approches sociales constructionnistes}

23 La perspective sociale constructionniste pourrait amener la recherche universitaire sur l'approche relationnelle du développement professionnel à une plus grande richesse créative (Blustein et al., 2004). La pensée sociale constructionniste facilite un entrelacs complexe de thèmes relationnels et professionnels, et ce d'une manière qualitativement différente de ce qui apparaît dans la plupart de nombreuses études traditionnelles, positivistes et post-positivistes (par exemple Hazan \& Shaver, 1990 ; O'Brien, 1996). Du point de vue social constructionniste, la connaissance se construit par des relations ancrées dans des contextes culturels, socio-économiques et sociopolitiques, et sont à l'opposé d'être les produits ou la possession d'un individu (Burr, 1995 ; Gergen, 1999). Ainsi la vision sociale constructionniste lie explicitement le comportement humain à l'interaction avec les autres, soulignant ce faisant le caractère central des relations dans le développement humain (Blustein et al., 2004 ; Schultheiss, Flum \& Blustein, 2003). Le but de l'investigation scientifique utilisant ce paradigme consiste alors à examiner comment on atteint des formes de connaissances ou d'identité dans l'interaction sociale (Burr, 1995).

Une autre implication importante de l'approche sociale constructionniste est qu'elle a conscience de l'existence de multiples aspects dans la façon qu'ont les gens de construire leur perception des relations et expériences de transition. De ce point de vue, l'identité professionnelle peut se conceptualiser comme construite et reconstruite à l'intérieur des relations et dans de multiples contextes. Ainsi, l'identité est continuellement émergente, reformée et redirigée dans des relations en constante évolution. Donc une compréhension beaucoup plus complexe et profonde de l'interface des relations et des transitions école-travail est possible en utilisant le paradigme 
social-constructionniste. Ce paradigme est bien adapté aussi à l'étude des relations et des transitions dans les diverses cultures et classes sociales. La façon dont ce paradigme est appliqué à la recherche est bien illustrée par les travaux de Young et de ses collègues (Young \& Valach, 2004 ; Young et al., 1997). Ces auteurs se sont en effet servi d'un modèle constructionniste pour proposer un point de vue convaincant sur les influences relationnelles complexes dont les adolescents et parents font l'expérience lors de leurs conversations sur les carrières.

\section{Perspectives théoriques et empiriques contemporaines sur les relations et les transitions scolaires et professionnelles}

Les travaux empiriques sur les transitions école-travail sont centrés sur deux ensembles de connaissances d'une importance primordiale : la définition de ce qu'est une transition adaptive, et l'identification d'antécédents ayant influencé les transitions réussies. Les transitions adaptives ont surtout été définies en utilisant des indicateurs économiques, mais d'autres mesures, plus orientées vers la psychologie, ont pris en compte l'adéquation entre les intérêts de l'individu et les exigences de l'emploi, ainsi que la satisfaction au travail (Blustein et al., 2000). On a identifié certains prédicteurs importants des transitions adaptives, dont le plus essentiel est l'accès aux structures de formation et d'orientation professionnelle (Bynner et al., 1997 ; Wilson, 1996). Bien que cette question puisse sembler difficile à traiter, les recherches présentent des résultats relativement cohérents en ce qui concerne la manière dont les individus peuvent maximiser leurs chances de vivre une transition adaptative et réussie (Blustein et al., 2000 ; Schoon \& Parsons, 2002). Par exemple, Schoon et Parsons (2002) ont obtenu des résultats suggérant que l'impact de la classe sociale des parents sur la réussite professionnelle de l'individu est médiatisée par sa réussite dans le domaine de la formation scolaire et professionnelle et par ses aspirations. Dans une revue de question portant sur les transitions de l'école au travail, Blustein et al. (2000) ont identifié quatre moyens par lesquels les jeunes se présentant sur le marché du travail peuvent modifier l'impact de leur classe sociale en renforçant leurs moyens de négocier cette transition efficacement. Ce sont: 1) développer des compétences à la fois dans les connaissances de base et dans les savoirs plus appliqués et pertinents pour un métier, 2) favoriser un ensemble de facteurs psychologiques comme l'initiative, la souplesse, la ténacité et le goût pour l'action, 3) entretenir un environnement relationnel de soutien actif, incluant la famille d'origine, des pairs soutenant et investis dans la relation, des enseignants, des psychologues du conseil, des collègues et supérieurs hiérarchiques, et 4) disposer d'un environnement de formation qui entretienne certains liens significatifs avec le monde du travail. La revue de question qui suit porte sur le troisième aspect décrit ci-dessus, à savoir le rôle des relations interpersonnelles en tant qu'antécédent important des transitions école-travail.

Bien que relativement peu d'études se soient centrées explicitement sur le contexte interpersonnel des transitions de l'école au travail, un corpus de connaissances commence à émerger. Way et Roseman (1996a, 1996b) ont présenté un ensemble de recherches instructives traitant du rôle que jouent les familles dans la préparation des individus au travail. Ces chercheurs ont conclu que ce sont la famille et son fonctionnement qui semblent médiatiser le succès d'une telle préparation au monde du travail. Mieux, ils ont clairement prouvé que la contribution du statut socio- 
économique au fait d'être prêt à travailler professionnellement dépend en partie de la façon dont la famille s'y prend pour préparer l'adolescent au monde du travail. D'après ce résultat, le type de famille qui a le plus de chances de promouvoir une transition école-travail facile peut être décrite comme proactive, c'est-à-dire des familles bien organisées, qui communiquent de manière ouverte et efficace, négocient positivement les conflits, recherchent les occasions de développement pour leurs membres, prennent leurs décisions démocratiquement, ont de la cohésion et le sentiment général de contrôler leur vie.

On trouve d'autres arguments empiriques en faveur de l'importance des relations interpersonnelles dans les transitions de l'école au travail dans un ensemble convaincant d'articles (Savickas, 2002b) paru dans un numéro spécial récent du Journal of Vocational Behavior. Tous ces articles présentent des éléments suggérant que, même si l'école et l'éducation à l'orientation présentent une certaine unité qui s'oppose au maintien des inégalités sociales, celles-ci subsistant, l'adolescent dispose de marges de manœuvre appréciables pour tirer profit des soutiens sociaux, de l'éducation parentale et de ses propres initiatives (Heckhausen, 2002). Par exemple, Heckhausen et Tomasik (2002) ont trouvé que, indépendamment des ressources dont dispose l'adolescent, les soutiens sociaux contribuaient de façon importante à une navigation réussie dans la transition école-travail en Allemagne et en Finlande. En accord avec ces résultats, Schoon et Parsons (2002) ont montré que l'influence parentale est un processus de médiation important dans deux échantillons britanniques dérivés de l'Étude nationale sur le développement de l'enfant de 1958 et de l'Étude de cohorte britannique de 1970. Plus spécifiquement, leurs résultats suggèrent que les aspirations des parents, c'est-à-dire en l'occurrence ce qu'ils espèrent pour leur enfant en ce qui concerne l'âge auquel il quittera l'école et la poursuite de sa formation, déterminent en partie ses aspirations et ses performances scolaires. En général, il semble qu'un degré élevé d'aspirations parentales exerce une forte influence sur la motivation des adolescents pour l'école et le développement chez eux d'aspirations professionnelles ambitieuses.

D'autres recherches européennes mettent aussi l'accent sur le rôle des relations interpersonnelles procurant du soutien. Reitzele, Vondracek et Silberseisen (1998) ont exploré les éléments essentiels du contexte socio-économique dans leur étude des jeunes entrant sur le marché du travail en ex-R.F.A. et ex-R.D.A. Se fondant sur un échantillon de jeunes adultes ne se destinant pas aux études supérieures dans la nouvelle Allemagne unifiée, ces chercheurs ont étudié l'organisation du temps lors des transitions école-travail. Le moment du premier choix d'orientation professionnelle, l'âge de fin d'études, de fin de formation professionnelle et celui de l'accession à l'indépendance financière ont été examinés. L'importance de comportements de soutien chez les parents, y compris leurs attentes d'une réussite scolaire et professionnelle, faisait partie des facteurs permettant de "prédire " le moment où se passeraient ces événements. D'autres chercheurs en Grande-Bretagne et en Allemagne (Evans \& Heinz, 1994) ont trouvé qu'outre le fait qu'ils avaient bien réussi leurs études, les individus bénéficiant du soutien social d'autres personnes étaient mieux équipés pour faire des transitions adaptives. Grâce à un ensemble de données tirées de l'Étude de la socialisation professionnelle chez les jeunes se présentant sur le marché du travail dans huit pays européens, Feij, Whitely, Peiro \& Taris (1995) ont identifié les relations avec les collègues et les supérieurs hiérarchiques comme étant des prédicteurs d'une transition adaptive vers le travail. 
29 Aux États-Unis, les élèves ont typiquement 18 ans lorsqu'ils intègrent le marché du travail directement après le lycée. Dans deux investigations exploratoires des transitions école-travail pour les travailleurs américains n'ayant pas fait d'études supérieures, Blustein et son équipe (Blustein et al., 2002 ; Blustein, Phillips, Jobin-Davis, Finkelberg \& Roarke, 1997) ont trouvé des éléments suggérant que le soutien émotionnel et instrumental des familles, d'autres personnes importantes et de psychologues du conseil facilitaient les transitions adaptives. En particulier, ils ont identifié l'importance des psychologues du conseil actifs et apportant une aide instrumentale (c'est-à-dire ceux qui proposaient une assistance tangible, par exemple dans la recherche d'un emploi) et l'importance d'un environnement de soutien et d'encouragement à la maison, à l'école et au travail. Dans une étude sur les lycéens américains en transition vers la vie active, Phillips et al. (2002) ont constaté que le soutien actif que les adultes apportent aux adolescents dans leur orientation vers le monde adulte facilite particulièrement les transitions adaptives. Les actions des adultes orientant les élèves vers le monde adulte allaient de l'apport d'un accès indirect au monde du travail grâce à leur expérience personnelle vécue (par exemple, faire profiter de son réseau de relations professionnelles, rechercher un emploi, proposer des conseils et des informations sur un emploi), jusqu'à l'aide instrumentale et émotionnelle (participer directement à la recherche de l'emploi, encourager...).

Dans d'autres études, l'intérêt du père et de la mère pour l'éducation de leur enfant (Bynner, 1998), leurs attentes, la discipline qu'ils exercent et leur implication dans l'accompagnement du jeune (Weisner, Vondracek, Capaldi \& Porfeli, 2003) apparaissent comme des prédicteurs importants des transitions adaptives. Plus spécifiquement, Bynner (1998), à partir d'échantillons tirés de l'Étude sur le développement de l'enfant, de 1958, et de l'Étude de cohorte britannique, de 1970, a présenté des arguments empiriques selon lesquels les enfants grandissant dans les familles des classes moyennes acquièrent un capital à la fois cognitif (évalué par des épreuves de lecture, écriture et calcul) et non-cognitif (en l'occurrence, l'intérêt porté par les parents à l'éducation de leur enfant) qui leur procure un certain niveau de protection contre les mauvaises conditions du marché du travail et les aident à obtenir et à garder un emploi. Weisner et al. (2003) ont étudié un échantillon d'hommes américains à risque (âgé de 23 à 24 ans), répartis en quatre groupes: (a) chômeurs de longue durée, (b) titulaires d'un emploi à court terme, (c) titulaires d'un emploi stable, et (d) étudiant ayant validé un premier cycle de quatre années d'études universitaires. Les auteurs ont constaté que ceux qui disaient avoir profité d'attentes élevées, de discipline et d'engagement chez leurs parents avaient plus de chances d'avoir une carrière réussie et productive (c'està-dire plus de chances d'aller à l'université, et moins de chances de subir le chômage de longue durée) que ceux qui n'en avaient pas profité.

31 Ayant pris acte des différences individuelles d'accès aux structures de formation et à l'emploi, les universitaires ont entrepris d'explorer la manière dont les individus peuvent agir pour maximiser leurs chances de réussite. Un intérêt naissant pour l'influence des relations interpersonnelles sur les transitions de l'école au travail est en train d'émerger de l'ensemble des travaux qui, antérieurement, avaient porté sur les effets de facteurs sociaux structurels et socio-économiques. Les résultats présentés plus haut soutiennent de façon relativement convergente l'idée selon laquelle la transition est facilitée par les soutiens sociaux et d'autres facteurs relationnels comme un degré élevé chez les parents d'attentes, d'aspirations, d'intérêt, d'engagement et d'exigence 
de discipline, ainsi qu'une structure familiale pro-active. Les bénéfices d'un réseau interpersonnel procurant un soutien social ne se limitent pas à celui fourni par la famille. Les résultats de plusieurs études suggèrent plutôt que des psychologues du conseil apportant un soutien tant émotionnel qu'instrumental, ou d'autres personnes significatives, ou encore des collègues et des supérieurs hiérarchiques, contribuent aussi à un type d'environnement relationnel de soutien actif qui aide au succès des transitions.

\section{Implications pour la Théorie, la Recherche et la Pratique}

Le concept d'âge de l'adulte en émergence convient bien pour cadrer théoriquement la période de développement au cours de laquelle la plupart des individus sont confrontés à des transitions scolaires et professionnelles et durant laquelle ils explorent des orientations de vie possibles. Ce cadre conceptuel est utile pour penser les défis que doivent affronter les individus pendant cette période de développement dynamique. Les recherches à venir pourraient s'en inspirer plus étroitement, en sorte de situer les transitions scolaires et professionnelles à l'intérieur d'un contexte de vie élargi. Comme les changements sociétaux, sociaux et économiques se produisent désormais à l'échelle du monde, il se peut que l'utilité de ce paradigme aille croissant à mesure que les jeunes de toutes les cultures font l'expérience de cette période prolongée d'exploration entre l'adolescence et l'âge adulte.

Il est possible que la recherche sur les transitions scolaires et professionnelles soit ellemême en transition, entre une approche privilégiant les facteurs sociaux structurels et une autre qui prend en compte aussi les caractéristiques du contexte rapproché, et en particulier du micro-milieu interpersonnel. L'état actuel des connaissances permet de repérer certaines perspectives de recherche et de pratique utiles pour l'avenir. L'étude de l'influence des relations familiales et d'autres relations interpersonnelles sur les transitions scolaires et professionnelles des jeunes issus de classes socioéconomiques défavorisées et qui ne s'inscrivent pas dans l'enseignement supérieur est un domaine de recherche prometteur. La carrière et les expériences professionnelles de ce groupe sont remarquablement absentes des théories traditionnelles de la psychologie de l'orientation et ont fait l'objet de peu de travaux empiriques. Il faudrait que, dans les prochaines avancées scientifiques, on examine de près la façon dont les facteurs socioéconomiques, culturels et historiques influencent la manière qu'ont les individus de négocier les transitions au cours de leur vie. Il serait important de considérer certains éléments spécifiques des transitions n'existant que dans certaines cultures ou à certaines époques, pour autant que les chercheurs du monde entier unissent leurs efforts pour mieux comprendre ce phénomène.

Dans les recherches futures, on pourrait aussi introduire d'autres paradigmes comme les approches sociales constructionnistes qui étudient les rapports entre les conduites humaines et les interactions avec autrui. Les méthodes qualitatives ayant une approche sociale constructionniste pourraient fournir un moyen de rapprocher les chercheurs des personnes qu'ils prétendent comprendre et encourager chez eux une pensée scientifique réfléchie. Il est possible d'obtenir des personnes participant aux recherches des récits et des histoires de vie, qui contribuent à forger de nouvelles théories et à 
mieux comprendre les rapports entre relations interpersonnelles et transitions du milieu scolaire au milieu professionnel.

Nous avons besoin d'une pratique de la psychologie du conseil qui soit plus globale, qui comprenne à la fois les problèmes personnels et ceux qui sont liés à la carrière professionnelle, et de modèles innovants en matière de conseil en développement de carrière, qui comportent les informations contextuelles essentielles et pertinentes pour la vie et les choix des bénéficiaires. Il conviendrait de mettre en œuvre de nouvelles pratiques fondées sur les relations interpersonnelles, semblables à celles qui sont mises en avant dans les publications relatives au conseil en développement de carrière (par exemple, Schultheiss, 2003), pour prendre en compte les multiples dimensions contextuelles des transitions scolaires et professionnelles. Les praticiens devraient s'efforcer de mieux comprendre les rapports entre les progrès dans la carrière et les expériences relationnelles des bénéficiaires. Les psychologues du conseil peuvent aider les bénéficiaires à se sentir partie prenante de leurs institutions de formation et d'orientation professionnelle à travers les relations avec leurs pairs, collègues et supérieurs hiérarchiques, de sorte qu'ils puissent leur demander aide et soutien. Ils peuvent aussi les aider à identifier les personnes pouvant leur prêter assistance. Par l'entretien de conseil, les bénéficiaires peuvent apprendre à rechercher le contact avec les autres, notamment face à des défis nouveaux et potentiellement angoissants concernant le champ professionnel, en particulier les transitions scolaires et professionnelles. Ainsi, les psychologues du conseil peuvent intervenir pour les aider à faire appel à des relations positives qui leur apportent un soutien ou facilitent le processus de transition d'une manière ou d'une autre. Par exemple, les personnes significatives peuvent servir de modèles de rôles efficaces, proposer des informations ou une assistance tangible, ou produire un impact sur d'importantes croyances personnelles. Les psychologues du conseil pourraient aussi aider les bénéficiaires à moins s'appuyer sur des interactions improductives, peu gratifiantes et inefficaces avec ceux qui les empêchent de progresser dans leur carrière, ce qui pourrait les rendre plus aptes à former et à utiliser des ressources relationnelles de façon plus productive et plus efficace pour faciliter leur progression de carrière.

\section{Conclusion et synthèse}

L'ensemble des travaux empiriques passés en revue fait ressortir l'importance des relations interpersonnelles en psychologie de l'orientation. Leur importance réside notamment dans les soutiens sociaux qu'elles procurent. Les familles, en particulier le père et la mère, présentent aussi d'autres conduites qui peuvent faciliter les activités d'orientation des adolescents et des adultes en émergence. Il s'agit en particulier de se montrer impliqué dans le développement du projet professionnel du jeune, d'exprimer des attentes à cet égard, de se montrer disponible pour une communication claire et ouverte dans un milieu familial uni. Les relations interpersonnelles intervenant dans le milieu scolaire, celui de formation professionnelle ou celui d'activité professionnelle apparaissent pouvoir produire des effets semblables aux effets des relations familiales sur la qualité des transitions du milieu de formation au milieu professionnel. Même si les réalités et contraintes socio-économiques exercent un effet considérable sur la négociation de cette transition, les facteurs contextuels tels que d'étroites relations interpersonnelles de soutien jouent sans aucun doute un rôle déterminant dans 
l'amélioration des résultats individuels. La négociation des transitions scolaires et professionnelles constitue un défi même pour les individus les mieux préparés. Le moment est venu de considérer sérieusement les facteurs relevant du contexte proche qui opèrent lors de l'émergence de l'adulte. Une perspective reconnaissant le rôle des relations interpersonnelles étroites avec les autres pourrait avoir une influence considérable lors des discussions sur la façon de mieux préparer les jeunes aux défis qui les attendent dans notre société mondialisée en pleine évolution.

\section{BIBLIOGRAPHIE}

Arnett, J. J. (2000). Emerging adulthood : A theory of development from the late teens through the twenties. American Psychologist, 55, 469-480.

Blustein, D. L. (1995). Toward a contextual perspective of the school-to-work transition : A reaction to Feij et al. Journal of Vocational Behavior, 46, 257-265.

Blustein, D. L. (2001). The interface of work and relationships : A critical knowledge base for $21^{\text {st }}$ century psychology. The Counseling Psychologist, 29, 179-192.

Blustein, D. L., Chaves, A. P., Diemer, M. A., Gallagher, L. A., Marshal, K. G., Sirin, S., \& Bhati, S. (2002). Voices of the forgotten half : The role of social class in the school-to-work transition. Journal of Counseling Psychology, 49, 311-323.

Blustein, D. L., Fama, L. D., White, S. F., Ketterson, T. U., Schaefer, B. M., Schwam, M. F., Sirin, S. R., \& Skau, M. (2001). A qualitative analysis of counseling case material : Listening to our clients. The Counseling Psychologist, 29, 240-258.

Blustein, D. L., Juntunen, C., \& Worthington, (2000). The school-to-work transition : Adjustment challenges of the forgotten half. In Brown \& Lent (Eds.), Handbook of Counseling Psychology ( $3^{\text {rd }}$ edition) (pp. 435-470). New York : Wiley.

Blustein, D. L., Phillips, S. D., Jobin-Davis, K., Finkelberg, S. L., \& Roarke, A. E. (1997). Atheorybuilding investigation of the school-to-work transition. The Counseling Psychologist, 25, 364-402.

Blustein, D., Prezioso, M., \& Schultheiss, D. (1995). Attachment theory and career development : Current status and future directions. The Counseling Psychologist, 23, 416-432.

Blustein, D. L., Schultheiss, D., \& Flum, H. (2004). Toward a relational perspective of the psychology of careers and working : A social constructionist analysis. Journal of Vocational Behavior, 64, 423-440.

Blustein, D., Walbridge, M., Friedlander, M., \& Palladino, D. (1991). Contributions of psychological separation and parental attachment to the career development process. Journal of Counseling Psychology, 38, 39-50.

Bowlby, J. (1988). A secure base : Parent-child attachment and healthy human development. NY : Basic Books.

Burr, V. (1995). An introduction to social constructionism. London : Routledge. 
Bynner, J. (1998). Education and family components of identity in the transition from school to work. International Journal of Behavioral Development, 22, 29-53.

Bynner, J. M., Ferri, E., \& Shepherd, P. (Eds.). (1997). Twenty-something in the 1990's: Getting on, getting by, getting nowhere. Brookfield, VT : Ashgate.

Chusid, H., \& Cochran, L. (1989). Meaning of career change from the perspective of family roles and dramas. Journal of Counseling Psychology, 36, 34-41.

Erikson, E. H. (1968). Identity: Youth and crisis. New York : Norton.

Evans, K., \& Furlong, A. (1996). Metaphors of youth transitions : niches, pathways, trajectories or navigations. In J. Bynner, L. Chisholm, \& A. Furlong (Eds.), Youth, citizenship and social change in a European context (pp. 17-24). Aldershot : Ashgate.

Evans, K., \& Heinz, W. R. (1994). Becoming adults in the 1990's. London : Anglo-German Foundation.

Feij, J. A., Whitely, W. T., Peiro, J. M., \& Taris, T. W. (1995). The development of career enhancing strategies and content innovation : A longitudinal study of new workers. Journal of Vocational Behavior, 46, 231-256.

Fisher, T. A., \& Stafford, M. E. (1999). Reliability and validity of the Career Influence Inventory : A pilot study. Journal of Career Assessment, 7, 187-202.

Gergen, K. J. (1999). An invitation to social constructionism. London : Sage.

Gilligan, C. (1982). In a different voice. Cambridge, MA : Harvard University Press.

Grotevant, H. D., \& Cooper, C. R. (1985). Patterns of interaction in family relationships and the development of identity exploration in adolescence. Child Development, 56, 415-428.

Grotevant, H. D., \& Cooper, C. R. (1986). Individuation in family relationships : A perspective on individual differences in the development of identity and role-taking skill in adolescence. Human Development, 29, 82-100.

Hall, D. T. and Associates. (1996). The career is dead-Long live the career : A relational approach to careers. San Francisco, CA : Jossey-Bass.

Harris, J. E., Moritzen, S. K., Robitschek, C., Imhoff, A., \& Lynch, J. L. A. (2001). The comparative contributions of congruence and social support in career outcomes. Career Development Quarterly, 49, 314-323.

Hazan, C., \& Shaver, P. R. (1990). Love and work : An attachment-theoretical perspective. Journal of Personality and Social Psychology, 59, 270-280.

Heckhausen, J. (2002). Introduction : Transition from school to work : Societal opportunities and individual agency. Journal of Vocational Behavior, 60, 173-177.

Heckhausen, J., \& Tomasik, M. J. (2002). Get an apprenticeship before school is out : How German adolescents adjust vocational aspirations when getting close to a developmental deadline. Journal of Vocational Behavior, 60, 199-219.

Jordan, J. V., Kaplan, A. G., Miller, J. B., Stiver, I. P., \& Surrey, J. L. (1991). Women's growth in connection: Writings from the Stone Center. New York : Guilford.

Josselson, R. (1992). The space between us : Exploring the dimensions of human relationships. San Francisco, CA : Jossey-Bass.

Juntunen, C. L., Barraclough, D. J., Broneck, C. J., Seibel, G. A., Winrow, S. A., \& Morin, P. M. (2001). American Indian perspectives on the career journey. Journal of Counseling Psychology, 48, 274-285. 
Kandel, D. B., \& Lesser, G. S. (1972). Youth in two worlds : US and Denmark. San Francisco : JosseyBass.

Kenny, M. E., Blustein, D. L., Chaves, A., Grossman, J. M., \& Gallagher, L. A. (2003). The role of perceived barriers and relational support in the educational and vocational lives of urban high school students. Journal of Counseling Psychology, 50, 142-155.

Kenny, M. E., \& Donaldson, G. A. (1992). The relationship of parental attachment and psychological separation to the adjustment of first-year college women. Journal of College Student Development, 33, 431-438.

Ketterson, T. U., \& Blustein, D. L. (1997). Attachment relationships and the career exploration process. The Career Development Quarterly, 46, 167-178.

Kohut, H. (1987). The restoration of the self. New York : International Universities Press.

Lapan, R. T., Hinkelman, J. M., Adams, A., \& Turner, S. (1999). Understanding rural adolescents' interests, values, and efficacy expectations. Journal of Career Development, 26, 107-124.

Lapsley, D. K., \& Edgerton, J. (2002). Separation-individuation, adult attachment style, and college adjustment. Journal of Counseling \& Development, 80, 484-492.

Larose, S., \& Boivin, M. (1998). Attachment to parents, social support expectations, and sociœmotional adjustment during the high school-college transition. Journal of Research on Adolescence, 8, 1-27.

Lent, R. W., Brown, S. D., Brenner, B., Chopra, S. B., Davis, T., Talleyrand, R., \& Suthakaran, V. (2001). The role of contextual supports and barriers in the choice of math/science educational options : A test of social cognitive hypotheses. Journal of Counseling Psychology, 48, 474-483.

Lent, R. W., Brown, S. D., \& Hackett, G. (1994). Toward a unifying social cognitive theory of career and academic interest, choice and performance. Journal of Vocational Behavior, 45, 79-122.

Lent, R. W., Brown, S. D., \& Hackett, G. (2000). Contextual supports and barriers to career choice : A social cognitive analysis. Journal of Counseling Psychology, 47, 36-49.

Lewis, T., Stone, J., Shipley, W., \& Madzar, S. (1998). The transition from school to work : An examination of the literature. Youth \& Society, 29, 259-292.

Lopez, F. G., \& Andrews, S. (1987). Career indecision : A family systems perspective. Journal of Counseling and Development, 65, 304-307.

Lopez, F. G., \& Gormley, B. (2002). Stability and change in adult attachment style over the firstyear college transition : Relations to self-confidence, coping, and distress patterns. Journal of Counseling Psychology, 49, 355-364.

Mattanah, J. F., Hancock, G., \& Brand, B. L. (2004). Parental attachment, separation-individuation, and college student adjustment : A structural equation analysis of mediational effects. Journal of Counseling Psychology, 51, 213-225.

McWhirter, E. H. Hackett, G., \& Bandalos, D. L. (1998). A causal model of the educational plans and career expectations of Mexican American high school girls. Journal of Counseling Psychology, 45, 166-181.

Miller, J. B., \& Stiver, I. (1997). The healing connection. Boston : Beacon Press.

Mitchell, S. A. (1988). Relational concepts in psychoanalysis. Cambridge, MA : Harvard University Press. 
O'Brien, K. (1996). The influence of psychological separation and parental attachment on the career development of adolescent women. Journal of Vocational Behavior, 48, 257-274.

Paa, H. K., \& McWhirter, E. H. (2000). Perceived influences on high school students' current career expectations. Career Development Quarterly, 49, 29-44.

Papini, D. R., \& Roggman, L. A. (1992). Adolescent perceived attachment to parents in relation to competence, depression, and anxiety : A longitudinal study. Journal of Early Adolescence, 12, 420-440.

Patton, W., \& McMahon, M. (1999). Career development and systems theory: A new relationship. Pacific Grove, CA : Brooks/Cole.

Phillips, S. D., Christopher-Sisk, E. K., \& Gravino, K. L. (2001). Making career decisions in a relational context. The Counseling Psychologist, 29, 193-213.

Phillips, S. D., Blustein, D. L., Jobin-Davis, K., \& White, S. F. (2002). Preparation for the school-towork transition : The views of high school students. Journal of vocational Behavior 61, 202-216.

Piaget, J. (1932). The moral judgment of the child. New York : Free Press.

Reitzle, M., Vondracek, F. W., \& Silbereisen, R. K. (1998). Timing of school-to-work transitions : A developmental-contextual perspective. International Journal of Behavioral Development, 22, 7-28.

Ryan, N. E., Solberg, V. S., \& Brown, S. D. (1996). Family dysfunction, parental attachment, and career search self-efficacy among community college students. Journal of Counseling Psychology, 43, 84-89.

Savickas, M. L. (1993). Career counseling in the postmodern era. Journal of Cognitive Psychotherapy, 7, 205-215.

Savickas, M. L. (2002a). Career construction : A developmental theory of vocational behavior. In D. Brown \& associates (Eds.), Career choice and development (4 $4^{\text {th }}$ ed., pp. 149-205). San Francisco : Jossey-Bass.

Savickas, M. L. (Ed.). (2002b). Transition from school to work [Special Issue]. Journal of Vocational Behavior, 60(2).

Schoon, L., \& Parsons, S. (2002). Commentary : At last research on career development in a developmental-contextual fashion. Journal of Vocational Behavior, 60, 310-319.

Schultheiss, D. (2003). A relational approach to career counseling: Theoretical integration and practical application. Journal of Counseling and Development, 81, 301-310.

Schultheiss, D. E. P., \& Blustein, D. L. (1994). Role of adolescent-parent relationships in college student development and adjustment. Journal of Counseling Psychology, 41, 248-255).

Schultheiss, D., Flum, H., \& Blustein, D. L. (2003, August). Relational discourse in the social construction of career and working. In R. Young \& A. Collins (Chairs), Social constructionism and career theory, research, and practice. Symposium conducted at the Annual Meeting of the American Psychological Association, Toronto, Canada.

Schultheiss, D., Kress, H., Manzi, A., \& Glasscock, J. (2001). Relational influences in career development : A qualitative inquiry. The Counseling Psychologist, 29, 214-239.

Schultheiss, D., Palma, T., Predragovich, K., \& Glasscock, J. (2002). Relational influences on career paths : Siblings in context. Journal of Counseling Psychology, 49, 302-310.

Scott, D. J., \& Church, A. T. (2001). Separation/attachment theory and career decidedness and commitment : Effects of parental divorce. Journal of Vocational Behavior, 58, 328-347. 
Silbereisen, R. K. (2002). Commentary : At last research on career development in a developmental-contextual fashion. Journal of Vocational Behavior, 60, 310-319.

Sullivan, H. S. (1953). The interpersonal theory of psychiatry. New York : Academic Press.

Tinsley, H. E. A. (1995). School to work transition. Journal of Vocational Behavior, 46, 229230.

Vondracek, F. W., Lerner, R. M., \& Schulenberg, J. E. (1986). Career development : A life-span developmental approach. Hillsdale, $\mathrm{NJ}$ : Erlbaum.

Wall, J., Covell, K., \& McIntyre, P. (1999). Implications of social supports for adolescents' education and career aspirations. Canadian Journal of Behavioral Science, 31, 63-71.

Way, W. L., \& Rossman, M. M. (1996a). Family contributions to adolescent readiness for school-towork transition. Journal of Vocational Education Research, 21(2), 5-33.

Way, W. L., \& Rossman, M. M. (1996b). Lessons from life's first teacher : The role of the family in adolescent and adult readiness for school-to-work transition (MDS-725). Berkeley, CA : National Center for Research in Vocational Education.

Weisner, M., Vondracek, F. W., Capaldi, D. M., \& Porfeli, E. (2003). Childhood and adolescent predictors of early adult career pathways. Journal of Vocational Behavior, 63, 305-328.

Whiston, S. C., \& Keller, B. K. (2004). The influence of the family of origin on career development : A review and analysis. Journal of Vocational Behavior, 32, 493-568.

Wilson, W. J. (1996). When work disappears : The world of the new urban poor. New York : Random House.

Winnicott, D. W. (1964). The child, the family, and the outside world. Middlesex, England : Penguin Books.

Young, R. A., \& Collin, A. (Eds.) (1992). Interpreting career: Hermeneutical studies of lives in context. Westport, CT : Praeger.

Young, R. A., \& Valach, L. (2004). The construction of career through goal-directed action. Journal of Vocational Behavior, 64, 499-514.

Young, R. A., Valach, L., Paseluikho, M. A., Dover, C., Matthes, G., E., Paproski, D., \& Sankey, A. (1997). The joint action of parents in conversation about career. Career Development Quarterly, 46, $72-86$.

Youniss, J. (1980). Parents and peers in social development. A Sullivan-Piaget perspective. Chicago : The University of Chicago Press.

Youniss, J., \& Smollar, J. (1985). Adolescent relations with mothers, fathers and friends.Chicago, IL : The University of Chicago Press.

\section{RÉSUMÉS}

Le fait que le développement de la carrière soit imbriqué dans le contexte plus large des rôles sociaux et des transitions caractérisant le cours de la vie est devenu un des centres d'intérêts remarquables en psychologie de l'orientation. Des travaux de plus en plus nombreux ont mis en évidence les rapports existant entre la carrière et la qualité des relations interpersonnelles dans la vie de l'individu (par exemple, Blustein, 2001 ; Blustein, Schultheiss \& Flum, 2004 ; Hall, 1996). L'intégration des dimensions relationnelles dans une conceptualisation élargie des transitions scolaires et professionnelles a permis l'élaboration d'un cadre conceptuel plus intégratif, qui 
prend en compte la complexité de la vie des individus, avec leurs contextes sociaux et culturels. L'article examine l'importance et la fonction des relations interpersonnelles dans la négociation par l'individu des tâches qui, dans sa carrière, le remettent en cause, telles que celles rencontrées lors des transitions scolaires et professionnelles. Les travaux théoriques et empiriques sont passés en revue et les implications pour la recherche et la pratique sont présentées.

The embedded nature of career development within the broader context of life roles and transitions has emerged as a prominent focus in the vocational psychology literature. A growing literature base points to the interconnectedness of career and the quality of relationships in one's life (e.g., Blustein, 2001 ; Blustein, Schultheiss, \& Flum, 2004 ; Hall, 1996). By integrating relational dimensions into a broad-based conceptualization of school and work transitions, a holistic conceptual framework emerges that reflects the complexity of people's lives embedded within social and cultural contexts. This paper will examine the importance and function of interpersonal connection in navigating challenging career tasks such as those encountered in school and work transitions. The theoretical and empirical literature will be reviewed, and implications for research and practice will be offered.

\section{INDEX}

Keywords : Attachment, Career, Interpersonal relationships, School, School-to-work transitions, Work transitions

Mots-clés : Attachement, Carrière, Relations interpersonnelles, Transitions de l'école au travail, Transitions professionnelles, Transitions scolaires

\section{AUTEURS}

\section{DONNA E. PALLADINO SCHULTHEISS}

Donna E. Palladino Schultheiss est « Associate Professor » dans le département de conseil, administration, supervision et formation des adultes, à l'Université de l'État de Cleveland Ohio, aux U.S.A. Le premier centre d'intérêt de ses recherches concerne la fonction adaptative des relations interpersonnelles et du contexte relationnel dans les progrès de la carrière.

La correspondance concernant cet article est à adresser à Donna E. Palladino Schultheiss, Department of Counseling, Administration, Supervision, and Adult Learning, Cleveland State University, 2121 Euclid Avenue, Cleveland, Ohio 44115, U.S.A., Phone : 216-687-5063, Fax : 216-687-5378. Toute correspondance électronique est à adresser à d.schultheiss@csuohio.edu 